\title{
Numerical modeling of the transition from low to high confinement in magnetically confined plasma
}

Rasmussen, Jens Juul; Nielsen, Anders Henry; Madsen, Jens; Naulin, Volker; Xu, G. S.

Published in:

Plasma Physics and Controlled Fusion

Link to article, DOI:

10.1088/0741-3335/58/1/014031

Publication date:

2016

Document Version

Peer reviewed version

Link back to DTU Orbit

Citation (APA):

Rasmussen, J. J., Nielsen, A. H., Madsen, J., Naulin, V., \& Xu, G. S. (2016). Numerical modeling of the transition from low to high confinement in magnetically confined plasma. Plasma Physics and Controlled Fusion, 58, 014031. https://doi.org/10.1088/0741-3335/58/1/014031

\section{General rights}

Copyright and moral rights for the publications made accessible in the public portal are retained by the authors and/or other copyright owners and it is a condition of accessing publications that users recognise and abide by the legal requirements associated with these rights.

- Users may download and print one copy of any publication from the public portal for the purpose of private study or research.

- You may not further distribute the material or use it for any profit-making activity or commercial gain

- You may freely distribute the URL identifying the publication in the public portal 


\title{
Numerical modeling of the transition from low to high confinement in magnetically confined plasma
}

\author{
J. Juul Rasmussen ${ }^{1}$, A.H. Nielsen ${ }^{1}$, J. Madsen ${ }^{1}$, V. Naulin ${ }^{1}$, \\ and G.S. $\mathrm{Xu}^{2}$ \\ ${ }^{1}$ PPFE, Dept. of Physics, Technical University of Denmark, Kgs. Lyngby, Denmark \\ ${ }^{2}$ Institute of Plasma Physics, Chinese Academy of Sciences, Hefei, People's Republic \\ of China
}

E-mail: jjra@fysik.dtu.dk

\begin{abstract}
.
The transition dynamics from low $(\mathrm{L})$ to high $(\mathrm{H})$ mode confinement in magnetically confined plasmas is investigated using a four-field drift fluid model - HESEL (Hot EdgeSol-Electrostatic). The model includes profile evolution and is solved in a $2 \mathrm{D}$ domain at the out-board mid-plane of a Tokamak including both open and closed field lines. The results reveal different types of L-H-like transitions in response to ramping up the input power by increasing the ion temperature in the edge region. For a fast rising input power we obtain an abrupt transition, and for a slow rising power we obtain a LI-H transition with an intermediate I-phase displaying limit cycle oscillations (LCO). The model recovers the power threshold for the L-H transition, the scaling of the threshold with the density and with the loss-rate in the SOL, indicating a decrease in power threshold when switching from single to double null configuration. The results hold promises for developing full predictive modeling of the L-H transition, which is an essential step in understanding and optimizing fusion devices.
\end{abstract}

Keywords: L-H transition, Fluid simulations, Braginskii equations, turbulence and flows Submitted to: Plasma Phys. Control. Fusion

\section{Introduction}

The understanding and prediction of the transition between the Low (L-mode) and High (H-mode) confinement mode is an outstanding and high priority issue in contemporary magnetic fusion research. Although the H-mode is routinely achieved in a multitude of magnetic confinement devices, since its first observation more than 30 years ago [1], the transition still lacks (full) theoretical explanation and predictive modeling. The L-mode is characterized by relatively flat pressure profiles with significant turbulent particle and energy transport across the Last Closed Flux Surface (LCFS) into the region of open field lines - the Scrape-Off Layer (SOL), see. e.g., [2, 3]. The H-mode is characterized by a pedestal of elevated pressure just inside the LCFS and a quiescent transport into the 
SOL leading to improved confinement [4]. The H-mode is of essential importance for the operation and success of ITER - the next generation international fusion experiment. To achieve the goal of ignition, ITER will rely on low power access to the H-mode. Additionally, it is presently envisaged that both DEMO and future fusion power plants will operate in the H-mode for optimized energy production.

Generally, the generation and sustainment of global - poloidal and toroidal - flows are observed to be key ingredients in the L-H transition, e.g., [5, 4, 6, 7]. The transition behaviour can vary from abrupt transitions to slow transitions with intermediate (I) phases $[8,4]$. Here, the I-phase refers to the transition phase between the L- and H-mode, characterized by strong quasi-periodic bursts of plasma into the SOL (the Iphase is also referred to as the dithering phase or limit-cycle oscillations (LCO)), see, e.g., $[9,10,11,12,13,14,15,16,17]$.

Recent experiments with advanced diagnostics provide detailed spatially and temporally resolved information about the L-H transition dynamics and it is now well established that the transition is connected with the build-up of Zonal Flows (ZF), sustained by turbulent Reynolds Stress (Re stress) and finally being sustained as Mean Flows (MF) supported by the steepened ion pressure gradient, e.g. [6, 7, 9, 10, 12, 14, 15]. The interaction between these players is complex and may in principle contain many elements, from electromagnetic perturbations to three-dimensional effects including details of the geometry.

Concurrent with the improved experimental diagnostics, new modeling approaches have been developed for describing the transition dynamics. The qualitative transition dynamics is well reproduced by heuristic zero- and more recently one-dimensional predator-prey type modeling, with one or two feedback loops acting on fast and slow time-scales, see, e.g., [18, 19, 20], but the quantitative connection to actual experimental parameters is absent in these models. This is to some extent remedied in the recent one-dimensional modeling by $\mathrm{Wu}$ et al [21] where the threshold power for the transition has been recovered together with simple scalings laws. In the work by Malkov et al [22] the earlier one dimensional models (e.g. [20]) are extended to consider different channels for the power input. It appears that the ion channel plays the main role in mediating the transition in agreement with recent observations [23].

Also several numerical simulations based on fluid type models have been attempted for modeling the transition dynamics, see, e.g., [24, 25, 26, 27]. They show clearly the transition and the key role of self-generated sheared flows for setting up the edge transport barrier, ETB. However, they do not provide a detailed comparison with experiments. Also, we consider the connection between the edge plasma region and the SOL across the last closed flux surface, LCFS, to be an essential issue in describing the L-H transition. This is included in the BOUT simulations [25].

The above mentioned results and the robustness of the $\mathrm{L}-\mathrm{H}$ transition indicates a basic mechanism that we believe is represented even in the interaction of the two-dimensional electrostatic turbulence with the self-consistently developing profiles, including the interaction between ion pressure and flow, specifically. Important is also 
the role of the Edge-SOL coupling anchoring initial gradients to the LCFS. Essential ingredients on the way towards improved understanding of the SOL dynamics have been to abandon the distinction between fluctuations and profiles and the usage of flux driven systems[28, 29, 3].

In this paper, we have applied the four field drift fluid model HESEL [30, 31] to investigate the L-H transition. The HESEL model includes the elements discussed above and in particularly it has realistic collisional and transport coefficients calculated from plasma parameters measured in experiments. The model is self-contained and consistently derived from the Braginskii equations [32]. It has no added features as, e.g., ensuring that the the poloidal flow relaxes to a pre-scribed value. The model, which is described in Sec. 2, applies to a wide range of parameters. In the present case we have used parameters corresponding to Asdex Upgrade and EAST sized Tokamaks. We have considered fast L-H transitions (Sec. 3.1) as well as slow transitions including an intermediate phases, L-I-H transition (sec. 3.2), and have additionally discussed scalings of the power threshold with density and SOL parallel connection length (Sec. 3.1). Finally, Sec 4 contains a discussion and concluding remarks.

\section{The HESEL Model}

\subsection{Model equations and set-up}

HESEL is a four-field, energy conserving fluid model, based on the Braginskii equations [32]. It describes interchange-driven, low-frequency turbulence in a plane perpendicular to the magnetic field at the outboard mid-plane of a Tokamak plasma. In the limit of constant ion pressure the model reduces to the ESEL model, e.g., [33, 34, 35, 36]. The HESEL model includes the transition from the confined region to the SOL and the full development of the profiles across the LCFS. The model is solved in a local slab geometry spanning the radial - poloidal, $(r, \theta)$, plane, with the inhomogeneous toroidal magnetic flux density approximated by $B(r)=B_{0} R /(R+a+r)$, where $a$, $R$, and $B_{0}$ are the minor, major radius of the torus, and the on-axis magnetic flux density, respectively. The direction of the magnetic field is into the plane implying that the ion and electron diamagnetic drift directions are in the positive and the negative poloidal direction, respectively. In the Bohm-normalization, the model equations read:

$$
\begin{aligned}
& \frac{d}{d t} n+n \mathcal{K}(\phi)-\mathcal{K}\left(p_{e}\right)=-\nabla \cdot\left(n \boldsymbol{u}_{R}\right)-\sigma(r) \frac{n}{\tau_{n}} \\
& \frac{d^{0}}{d t} w+\left\{\nabla \phi, \nabla p_{i}\right\}-\mathcal{K}\left(p_{e}+p_{i}\right) \\
& =\eta \nabla^{2} w-\sigma(r)\left[\frac{w}{\tau_{w}}+\frac{\rho_{s}}{L_{\|}}\left[1-\exp \left(\phi_{m}-\frac{\langle\phi\rangle}{\left\langle T_{e}\right\rangle}\right)\right]\right] \equiv \Lambda_{w} \\
& \frac{3}{2} \frac{d}{d t} p_{e}+\frac{5}{2} p_{e} \mathcal{K}(\phi)-\frac{5}{2} \mathcal{K}\left(\frac{p_{e}^{2}}{n}\right) \\
& =\nabla \cdot\left(\chi_{e \perp} \nabla T_{e}\right)-\frac{5}{2} \nabla \cdot\left(p_{e} \boldsymbol{u}_{R}\right)-\boldsymbol{u}_{R} \cdot \nabla p_{i}-\frac{3 m_{e}}{m_{i}} \nu_{e i}\left(p_{e}-p_{i}\right)-\sigma(r) \frac{p_{e}}{\tau_{p_{e}}},
\end{aligned}
$$


Numerical modeling of the transition from low to high confinement in magnetically confined plasma4

$$
\begin{aligned}
& \frac{3}{2} \frac{d}{d t} p_{i}+\frac{5}{2} p_{i} \mathcal{K}(\phi)+\frac{5}{2} \mathcal{K}\left(\frac{p_{i}^{2}}{n}\right)-p_{i} \mathcal{K}\left(p_{e}+p_{i}\right) \\
& =\nabla \cdot\left(\chi_{i \perp} \nabla T_{i}\right)-\frac{5}{2} \nabla \cdot\left(p_{i} \boldsymbol{u}_{R}\right)+\boldsymbol{u}_{R} \cdot \nabla p_{i}+\frac{3 m_{e}}{m_{i}} \nu_{e i}\left(p_{e}-p_{i}\right)-\sigma(r) \frac{p_{i}}{\tau_{p_{i}}}+p_{i} \Lambda_{w},
\end{aligned}
$$

where time is normalized with the ion cyclotron period, $\omega_{c i}^{-1}$, and length with $\rho_{s}=c_{s 0} / \omega_{c i}$ $\left(c_{s 0}=\sqrt{T_{e 0} / m_{i}}\right)$, here $T_{e 0}$ is the reference electron temperature. The electrostatic potential, $\phi$, is normalized with $T_{e 0} / e$, particle density, $n$, with a reference density, $n_{0}$, temperatures, $T_{e, i} \equiv p_{e, i} / n$ with reference temperatures $T_{e 0, i 0}$, and pressures $p_{e, i}$ with the reference pressures $n_{0} T_{e 0, i 0} . \quad w=\nabla_{\perp}^{2} \phi+\nabla_{\perp}^{2} p_{i}$ is the generalized vorticity. The function, $\sigma(r)=\left(1+\tanh \left[0.5\left(r-r_{S E P}\right)\right]\right) / 2$, describes the transition from closed to open magnetic field lines, $r_{S E P}$ is the position of the LCFS. $\Lambda_{w}$ is short hand notation for the right hand side of Eq. 2. Material derivatives and the magnetic field curvature operator are defined as $\frac{d}{d t}=\frac{\partial}{\partial t}+\frac{1}{B} \hat{\boldsymbol{z}} \times \nabla \phi \cdot \nabla$ and $\mathcal{K}=\nabla\left(\frac{1}{B}\right) \cdot \hat{\boldsymbol{z}} \times \nabla$, respectively, except in the generalized vorticity equation where the material derivative $\frac{d^{0}}{d t}$ is taken with a constant magnetic field. Friction forces enter through the drift velocity $\boldsymbol{u}_{R}=-D\left(1+T_{i} / T_{e}\right) \nabla \ln n$, where $D=\left(1+1.6(R / a) q^{2}\right) \nu_{e i} \rho_{e}^{2}$ is the neoclassical Pfirsch-Schlüter diffusion coefficient [34]. Here, $q$ is the safety factor, $\nu_{e i}$ and $\rho_{e}$ are normalized electron-ion collision frequency and electron Larmor radius, respectively. $\eta=0.3\left(1+1.6(R / a) q^{2}\right) \nu_{i i} \rho_{i}^{2}$ is the perpendicular viscosity coefficient due to ion-ion collisions including neo-classical corrections, with $\nu_{i i}$ and $\rho_{i}$ normalized ion-ion collision frequency and ion Larmor radius, respectively. $\chi_{e \perp}=14 / 3 D$ and $\chi_{i \perp}=20 / 3 \eta$ are the normalized neo-classical, perpendicular electron and ion heat conduction coefficients, respectively. Losses due to advection along magnetic field lines in the SOL region $\left(r>r_{S E P}\right)$ are represented by the damping rates $\tau_{n}^{-1}, \tau_{w}^{-1}$, and $\tau_{p_{i}}^{-1}$, and parallel electron heat conduction by $\tau_{T_{e}}^{-1}$, where $\tau_{n}=\tau_{w}=L_{\|} / 6 M c_{s 0}$, $\tau_{p i}=2 / 9 \tau_{n}$, and $\tau_{p_{e}}=\left(\nu_{e i} m_{e} L_{\|}^{2}\right) /\left(6.32 T_{e 0}\right) ; M=0.5$ is the parallel Mach number and $L_{\|}$is the connection length, i.e., the distance from the outboard mid-plane to the divertor plate along the magnetic field lines. The effects of sheath currents at material surfaces on which magnetic fields lines in the SOL terminates, is approximated by a sheath dissipation term entering the vorticity Eq. (2), where $\phi_{m} \equiv \ln \left(\sqrt{m_{i} / \pi m_{e}}\right)$ is the Bohm potential, and $\langle\cdot\rangle$ denotes a poloidal average.

The generalized vorticity, $w$, is the manifestation of the polarization current in the model and describes charge separation due to the inertia in the ion response to changes in the $\boldsymbol{E} \times \boldsymbol{B}$ and diamagnetic drifts, i.e., $\hat{b} \cdot \nabla \times\left(\boldsymbol{v}_{E}+\boldsymbol{v}_{d i}\right) \propto \nabla_{\perp}^{2} \phi+\nabla_{\perp}^{2} p_{i} \equiv w$, where $\boldsymbol{v}_{E}$ and $\boldsymbol{v}_{d i}$ are $\boldsymbol{E} \times \boldsymbol{B}$ and ion diamagnetic velocities, respectively, see, e.g., [37]. The generalized vorticity is responsible for driving the MF related to the pressure gradient and is essential for setting up the edge transport barrier supporting the pressure pedestal in the H-mode. Simulations with only the $\boldsymbol{E} \times \boldsymbol{B}$ fluid vorticity, $\nabla_{\perp}^{2} \phi$, do not reveal a L-H transition.

In the inner part of the closed field line region the pressures and particle density are forced towards prescribed profiles resembling empirical profiles in the edge region of toroidal plasmas. The forced profiles are the dominant pressure and particle density 
sources in the system. They also remove the "unphysical" instabilities that may be generated near the wall due to sharp gradients. We apply Dirichlet boundary conditions (BC) in the radial direction for pressures, density and vorticity on the inner radial boundary and Neumann BC on the outer boundary, while the potential has Neumann $\mathrm{BC}\left(\partial_{r} \phi=0\right)$ on the inner boundary and Dirichlet BC $(\phi=0)$ on the outer boundary. The prescribed values of pressure and particle density on the the inner radial boundary are in agreement with the forced profiles in the inner part of the edge region, while the vorticity is set to zero. In the poloidal direction we have periodic BC. The model is solved by employing a finite difference method with a symmetry, energy, and enstrophy conserving discretization of the nonlinear advection terms and an explicit third-order stiffly stable time integrator with diffusive terms treated implicitly using operator splitting [38].

Before describing the results we briefly discuss the properties of the linear modes included in the model. The 2D slab geometry includes the Ideal Interchange Mode, IIM, modified by finite ion temperature effects and dissipation terms. This mode has a maximum linear growth rate, $\gamma$, and transport towards small perpendicular wave

numbers, $k_{\perp}=\sqrt{k_{r}^{2}+k_{\theta}^{2}}$. However, we find that $\gamma \propto k_{\theta}$ for a finite, fixed radial wave number, $k_{r}$. Thus, the growth rate will be maximum for finite values of $k_{\theta}$ and vanish for $k_{\theta} \rightarrow 0$. In a realistic Tokamak geometry it is clear that this $2 \mathrm{D}$ mode will be strongly modified by the sheared magnetic field and the influence of finite parallel wavelength must be accounted for, i.e., drift-wave dynamics should be included. The relevant modes appear to be of the Resistive Ballooning Mode, RBM, type, e.g., [39]. We believe, however, that the nature of the linear modes is not essential for the gross properties of the transition - the essential point is the interplay between the turbulence and the profiles, which the model allow for. We should note that the quantitative differences in the transport of the different modes might have an impact on the quantitative value of the L-H threshold power, see, e.g., Ref. [40] for the difference in transport mediated by drift waves and interchange modes, respectively. Additionally, the RBM have the largest transport at the outboard mid-plane due to the ballooning effect, and we may argue that in a slab at the outboard mid-plane the IIM will locally mimic the RBM.

\subsection{Simulation parameters}

For the results presented in the present paper we applied parameters typical for medium sized tokamaks of the Asdex Upgrade and EAST type. However, the applicability of the model is not restricted to these cases. The model may be applied to a wide span of parameter values. We should emphasize that the collision frequencies and the parallel damping coefficients are consistently calculated from the actual plasma parameters, which is a unique feature of the model.

To be specific we have used the parameters for the plasma in EAST shot 41362 during the $2012 \mathrm{~L}-\mathrm{H}$ transition campaign, see e.g., [10, 11]. Plasma parameters entering the collision terms in the model are set to the measured values at the LCFS: Particle 
density $n_{0}=1.5 \times 10^{19} \mathrm{~m}^{-3}$, electron temperature $T_{e 0}=20 \mathrm{eV}$, and ion temperature $T_{i 0}=20 \mathrm{eV}$ (base value). Further parameters are distance from the LCFS to the limiter shadow $\Delta_{S O L}=2.4 \mathrm{~cm}$ magnetic field on axis $B_{0}=2.0 \mathrm{~T}$, safety factor $q_{95}=4.5$, major radius $R=2.0 \mathrm{~m}$, and minor radius $a=0.5 \mathrm{~m}$. With these parameters we obtain the following values for the Bohm normalized transport coefficients: $D=0.0019$, providing a particle diffusion coefficient $D_{n}=0.0038, \eta=0.018, \chi_{e \perp}=0.0089$, and $\chi_{i \perp}=0.12$.

We model the power input by ramping-up the ion temperature at the inner boundary, which leads to an increased ion heat flux. In that respect the model is considered as flux driven. The prescribed ion pressure profile in the vicinity of the inner boundary follows the temperature at the wall. The reference ion temperature projected to the LCFS is changed accordingly as:

$$
T_{i 0}(t)=T_{i 0}(0)+\left(T_{i 0}\left(t_{\max }\right)-T_{i 0}(0)\right) \sin \left(\frac{\pi}{2} \frac{t}{t_{\max }}\right)
$$

where $T_{i 0}(0)=20 \mathrm{eV}$ and for most of the results $T_{i 0}\left(t_{\max }\right)=60 \mathrm{eV} . t_{\max }$ is the ramp-up time. The neo-classical transport coefficients and parallel loss rates - calculated from the reference parameters at the LCFS - are adjusted consistently during the variation of the ion temperature.

\section{Results}

The L-H transitions observed in the simulations are robust and have been obtained for a broad range of parameters. Different types of transitions have been observed ranging from fast $(\mu \mathrm{s})$ transitions to slow (ms) transitions with a clear intermediate I-phase. Also L-I-L transitions are observed when the power input is slightly lower than the critical power.

\subsection{Fast transition}

First we consider a fast ramp-up with a one-step L-H transition. In Fig. 1 we show a typical example of the $\mathrm{L}-\mathrm{H}$ transition with $t_{\max }=2.6 \mathrm{~ms}$. In the bottom panel (e) we show the evolution of the ion temperature according to Eq. 5. The two upper panels display the temporal evolution of of radial profiles of the electron (a) and ion (b) pressure, respectively. The profiles are obtained by averaging the pressures over the poloidal direction. The pressure difference across the LCFS is depicted in (c). While we in (d) show the integrated energy flux across the LCFS. We take this as a proxy for the $D_{\alpha}$-signal, which is often used to characterize a confinement transition in experiments, see, e.g., [4]. In experiments the $D_{\alpha}$ emission located in the divertor region is interpreted as a measure for the amount of hot plasma transported across the LCFS into the SOL at the outboard mid-plane. This plasma subsequently flows along the field lines to the divertor region, where it leads to an enhanced excitation of the neutral D atoms. Thus, the transition to the $\mathrm{H}$-mode is marked by a significant reduction in the $D_{\alpha}$ emission. Figure 1 reveals the characteristic features of the L-H transition. As the ion heat flux 


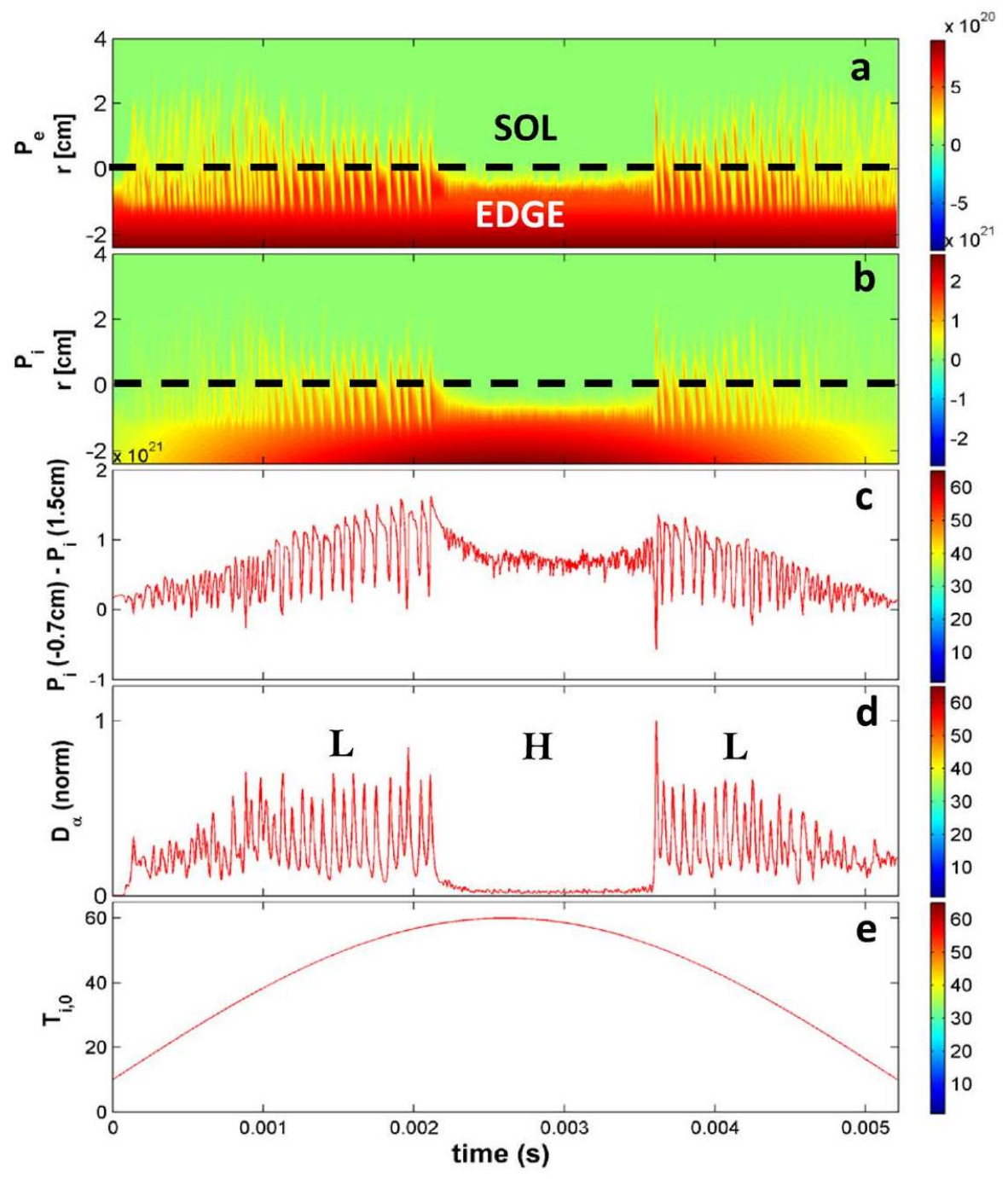

Figure 1. Typical L-H-L transition with input power ramped up by raising the ion temperature at the inner boundary. a) Time evolution of the electron pressure profile across LCFS at $(r=0)$ marked by the dashed line. b) The ion pressure profile. c) The difference in the ion pressure between radial positions $r=-0,7 \mathrm{~cm}$ and $r=1,5$ $\mathrm{cm}$. d) The integrated particle loss term as a proxy for the $D_{\alpha}$ emission from the outer divertor region. e) The reference ion temperature as function of time. Note that the frames $\mathrm{a}$ and $\mathrm{b}$ do not contain the inner region, where profiles are forced.

is ramped up by increasing the ion temperature the plasma abruptly changes from the L-mode state with intermittent fluctuations and significantly increased transport across the LCFS into a quiescent phase with an almost fixed pressure difference (c) and vanishing turbulent transport (d) across the LCFS. We also note a hysteresis when ramping down the ion temperature, the H-L transition appears at a significantly lower temperature than the $\mathrm{L}-\mathrm{H}$ transitions.

The profiles just before and after the transition of density, ion and electron pressure, poloidal $\boldsymbol{E} \times \boldsymbol{B}$ velocity, $V_{p}$, and ion and electron energy flux densities $\left(Q_{i, e} \equiv\left\langle\tilde{p}_{i, e} \tilde{v}_{r}\right\rangle\right)$ are shown in Fig. 2. The profiles are obtained by averaging over the poloidal direction 
Numerical modeling of the transition from low to high confinement in magnetically confined plasma8
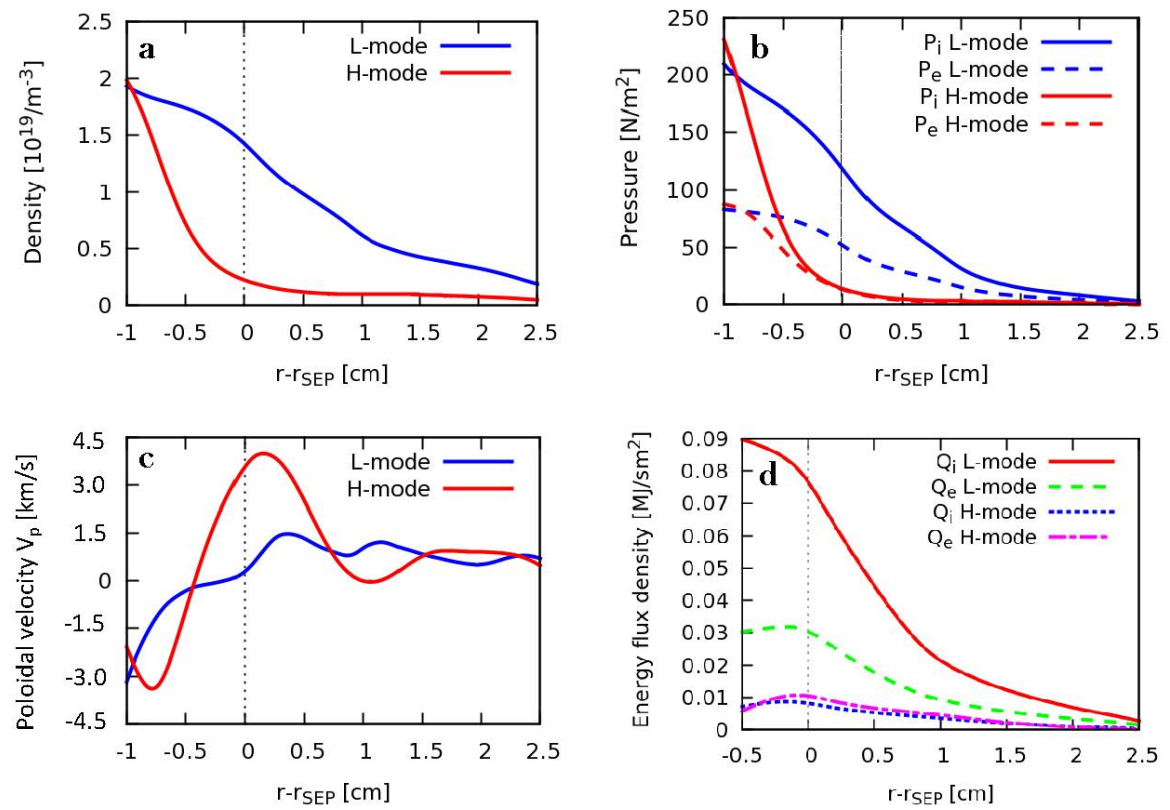

Figure 2. Profiles just before and after the L-H transition. a) Density profile b) The ion and electron pressure profile. c) The poloidal $\boldsymbol{E} \times \boldsymbol{B}$ velocity profile. d) The ion and electron energy flux density mediated by the turbulent fluctuations. Note that the plotted region do not contain the inner region, where profiles are forced.

and particularly for the L-mode over a time interval covering several oscillation periods. In the L-mode the density (Fig. 2a) and pressure profiles ( Fig. 2b) are broad and stretch across the LCFS, while in the H-mode we observe sharp gradients in both density and pressure inside the LCFS and a limited penetration into the SOL. We should note that the temperature profiles $T_{e, i}=p_{e, i} / n$ are more shallow than the pressure and density profiles. The poloidal $\boldsymbol{E} \times \boldsymbol{B}$ velocity $-V_{p}$ - profile (Fig. 2c) clearly shows the formation of a strongly sheared flow with a maximum velocity around $3.6 \mathrm{~km} / \mathrm{s}$ in the electron diamagnetic direction, which corresponds to $0.12 c_{s 0}$ for the present parameters. This is the edge transport barrier, ETB, sustaining the H-mode. The poloidal mean flow flow is supported by the steep ion pressure gradient, and the dynamics of the generalized vorticity $w=\nabla_{\perp}^{2} \phi+\nabla_{\perp}^{2} p_{i}$ is providing the connection between the radial electric field and the ion pressure gradient. We note that a stationary solution for the generalized vorticity equation, Eq. 2 , is $\nabla_{\perp}^{2} \phi+\nabla_{\perp}^{2} p_{i}=0$, this may be integrated to yield $E_{r}=-\partial_{r} \phi(r)=\partial_{r} p_{i}(r)+$ const.. For the case in Fig. 2, we note that the maximum $\boldsymbol{E} \times \boldsymbol{B}$ velocity of $\left|V_{p}\right|=3.6 \mathrm{~km} / \mathrm{s}$, is close to the maximum ion diamagnetic velocity $\approx 5.7 \mathrm{~km} / \mathrm{s}$

Generally, the $\boldsymbol{E} \times \boldsymbol{B}$-flow setting up the transport barrier, ETB, in the H-mode is argued to be determined by the radial force balance, which is assumed to hold in the quasi-stationary equilibrium of the H-mode, e.g., $[5,6]$. The radial force balance reads (in unnormalized units):

$$
E_{r} \approx \frac{\partial_{r} p_{i}}{n e}+v_{\theta} B
$$


Numerical modeling of the transition from low to high confinement in magnetically confined plasma9

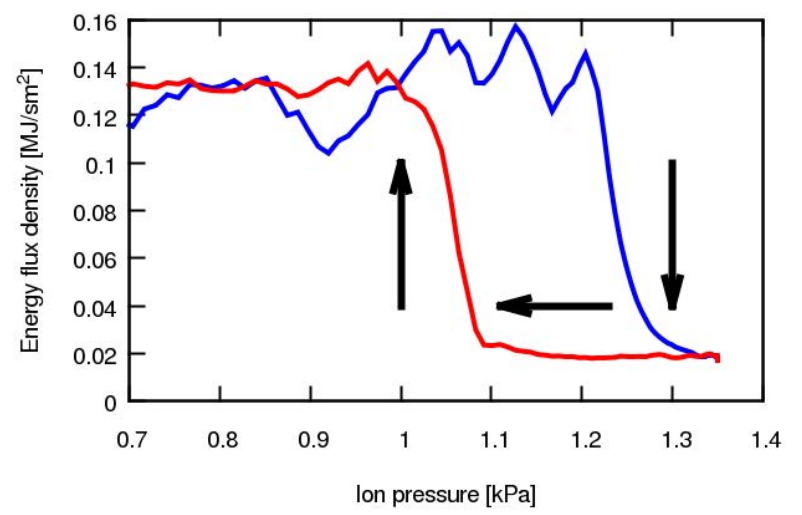

Figure 3. HESEL simulations displaying the total turbulent energy flux density across the LCFS as a function of the ion pressure at the LCFS during a transition.
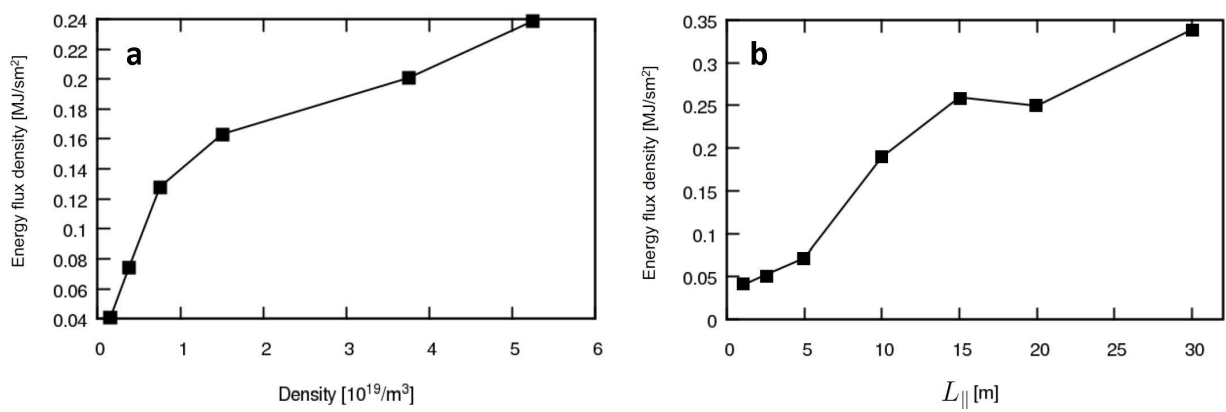

Figure 4. HESEL simulations displaying threshold energy flux density across the LCFS at the transition for different densities (a) and for varying connection length (b). The connection length determines a characteristic parallel advection loss time, $\tau \propto L_{\|} / c_{s 0}$.

for the given geometry, where $B$ is the magnitude of the toroidal magnetic flux, the magnetic field points into the $x-y$ plane, i.e., in the negative $z$-direction. (In our model there is no poloidal B-field). $E_{r}$ is the radial electric field and $v_{\theta}$ is the poloidal velocity. We should emphasize that the radial force balance is inherently embedded in the drift fluid model, where the ion-velocities to the lowest order are actually determined by the radial force balance. Thus, no further information on the flow dynamics may be deduced from Eq. 6 .

Finally, the energy flux density profiles (Fig. 2d) clearly show the dominating ion energy flux just before the transition. After the transition the turbulent driven fluxes (shown in the figure) are significantly reduced and the ion and electron energy fluxes are comparable, they clearly show the effect of the ETB.

To investigate the threshold power for the L-H transition, we have plotted in Fig. 3 the evolution of the (total) energy flux density across the LCFS as function of the ion pressure at the LCFS during an L-H transition and the $\mathrm{H}-\mathrm{L}$ back-transition for the case shown in Fig. 1. As the ion pressure is decreased a clear ion pressure hysteresis is observed with a $\mathrm{H}-\mathrm{L}$ transition occurring at a lower ion pressure compared to the L-H threshold - as is also observed in Fig. 1. The reference particle density is kept constant 
throughout the simulation. After the H-L transition the energy flux density reverts to the same level as before the transition. Hysteresis in different quantities has often been observed in experiments [4]. However, the hysteresis depends on which quantity one considers. From Fig. 3 we observe that the L-H and the H-L transition appears at the same value of the energy flux density. Thus, following the variation of the energy flux density one would not expect to observe a hysteresis.

The total heat flux across the LCFS - or the total loss power - at the L-H transition is estimated from the HESEL simulations to 1.2 MW, which is equivalent to the power threshold for the transition using the standard definition. The loss power has been obtained from the assumption that the heat flux crossing the LCFS has an asymmetric distribution and originates from a region 30 degree stretching poloidally above and below the outboard mid-plane. This threshold power is in agreement with the estimated measured threshold input power of around 1.0 MW for these particular parameters in EAST [10].

We have also investigated the general scalings of the L-H transition threshold power. Figure 4a shows a series of HESEL simulations for different reference particle densities $n_{0}$. The transition threshold power increases at increasing particle density, as generally observed in the high density branch of the L-H transition threshold power [4]. We have, however, not found the experimentally observed fast increase of the L-H power threshold at lower densities, leading to a well-defined density with minimal power threshold. In these experiments electrons are dominantly heated centrally. However, recently Ryter et al. [23] demonstrated that the ion heat channel plays the key role in the L-H transition and the ramp-up of the ion energy flux across the LCFS depends on the energy coupling between electrons and ions over the whole plasma volume. This coupling is weaker at low densities which may explain the increase in the electron power threshold at low density. When the power input is directly through the ions one should not expect to find a roll-over of the threshold power at a specific density.

Figure $4 \mathrm{~b}$ shows the total energy flux density across the LCFS as function of the parallel connection length $L_{\|}$, which is related to the characteristic parallel advection loss time, $\tau \propto L_{\|} / c_{s 0}$. As $L_{\|}$decreases we observed a nearly linear decrease in the threshold power for L-H transition. This agrees with experimental observations, where it is well established that there is significant reduction of the power threshold when operating in a double null divertor configuration as compared with the single null configuration. This was first observed in MAST [41] and later confirmed in several other devices, e.g., Asdex Upgrade [42], NSTX [43], and EAST [44]. Also, recent observations from TCV report a reduction in power threshold as the X-point is moved downward thus decreasing $L_{\|}$ [45]. Note that for shorter connection length the SOL will be emptied faster of plasma - the SOL acts as an anchor point for the pressure gradient in the edge and an empty SOL favors steeper gradients. Thus, the SOL conditions and the divertor configuration play essential roles for the L-H transition and the threshold scaling, see also [46, 47]. Therefore, any predictive modeling of the $\mathrm{L}-\mathrm{H}$ transition must include the transition between closed and open field lines and a proper SOL description. 


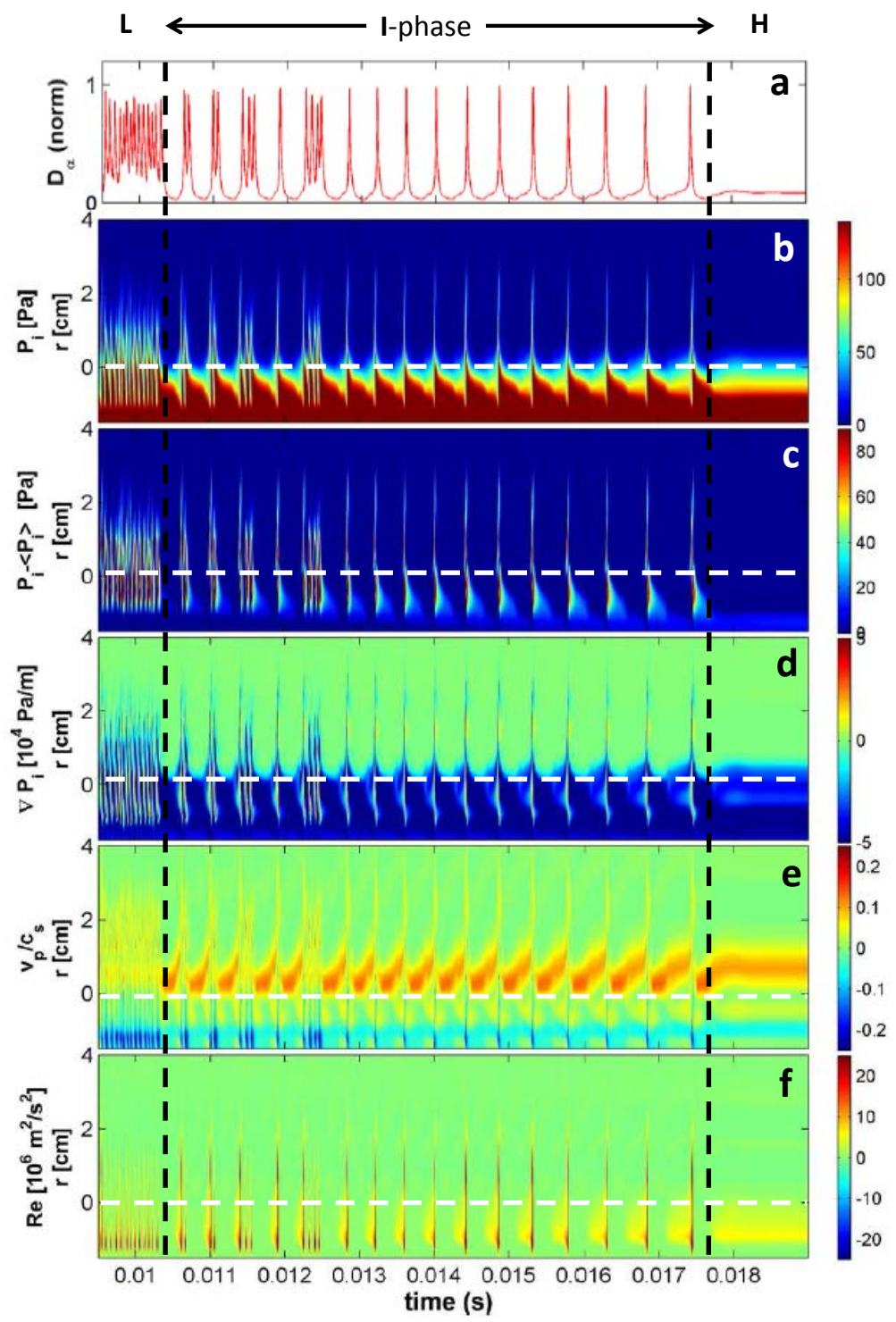

Figure 5. HESEL simulation for the dithering L-I-H transition. a) integrated parallel particle loss term as a proxy to divertor $D_{\alpha}$, b) ion pressure profile evolution. c) ion pressure profile fluctuations d) ion pressure gradient profile e) poloidal $E \times B$ flow profile f) Reynolds Stress derived from $E \times B$ velocities. The horizontal dashed lines mark the LCFS $(r=0)$. The frames b-f do not contain the inner region, where profiles are forced.

\subsection{The slow transition: $L-I-H$ transition}

We consider an example of a slow transition with an intermediate dithering phase with Limit Cycle Oscillations (LCO). The characteristic ramp-up time is approximately 10 times longer than the case considered in Fig. 1. In Fig. 5 we show the evolution through the transition. We clearly identify the L-mode and the H-mode at the beginning and end in all the displayed panels. Between these two states we observe the I-phase with a 
number of LCO's, where bursts of plasma are quasi-periodically being ejected from the edge region into the SOL, see Fig. 5a, with the evolution of the flux across the LCFS as a proxy for the $D_{\alpha}$ emission. This leads to an increasing fluctuation level, Fig. 5c, and breakdown of the pressure gradient, Figs. 5b and d. The number of LCO's is related to the power ramp-up rate, a slower or faster ramp-up rate will result in more or less LCO-periods, respectively. The periods of the LCO's are slightly increasing through the I-phase, with a frequency varying from $2 \mathrm{kHz}$ to $1.5 \mathrm{kHz}$ when approaching the I-H transition. This is commonly observed in the experiments, e.g., [12, 14, 15, 10], with the I-phase and also in the 1D, e.g., [20, 21] and even 0D, e.g., [18], modelings.

In a separate paper [48] we have made a detailed comparison of the simulations with the experimentally observed L-I-H transition in EAST shot 41362 and found close agreement in several respects. The comparisons were facilitated by a synthetic GPI diagnostics implemented in HESEL to resemble the EAST GPI diagnostic, [49].

As described above the I-phase appears as a series of quiet periods supported by a significant poloidal flow as observed in Fig. 5e. These quiet periods are quasi-periodically interrupted by a break down of the flow and large bursts of plasma reaching far into the SOL. The flow builds up again mediated by the Re-stress depicted in Fig. 5f. One may consider the I-phase as a series of attempts to enter the H-mode, which temporary prevails during the quiet phases. However, these H-modes cannot be sustained before a sufficiently strong poloidal flow is created due to the sharp ion pressure gradient. This flow appears to be mainly sustained by the pressure gradient as discussed in subsection 3.1 , where we noted that the generalized vorticity may consistently lead to this quasiequilibrium state. In Fig. 6 we show the detailed evolution during the the last dithering cycle from Fig. 5. All the cycles in the I-phase have an overall similar appearance, except that this last cycle stays in the H-mode. We identify three distinct phases, separated by the vertical lines in Fig. 6. The first is quiescent with nearly no fluctuations and a sheared poloidal flow forming a temporary transport barrier. It lasts until $T \sim 17.42$ ms, where significant fluctuations grow up and are dominating the dynamics. The pressure gradient and the poloidal flow is expelled from the edge deep into the SOL see Fig. 6a, b, and c. In the final phase, from $T \sim 17.46 \mathrm{~ms}$ the fluctuations and the enhanced transport into the SOL die out. The SOL is emptied of plasma and finally the pressure gradient across the LCFS re-establishes. The disappearance of the turbulent fluctuations is accompanied by a strongly enhanced Re-stress and Reynolds work $W_{\text {Re }}$ (Fig. 6d and e)

$$
W_{R e} \equiv\left\langle v_{r} v_{p}\right\rangle \frac{\partial V_{p}}{\partial r}
$$

which adds to re-establishing the poloidal $\boldsymbol{E} \times \boldsymbol{B}$ flow $V_{p} \equiv\left\langle v_{p}\right\rangle . v_{r}, v_{p}$ are radial and poloidal velocity components, respectively. The generated poloidal flow, Fig. 6b, is later in parts sustained by the ion pressure gradient, Fig. 6c, induced mean flow (MF). But if the MF is not strong enough to sustain the barrier, it will lead towards the next burst and closing the cycle. If, on the other hand, the MF, grows strong enough, this burst never occurs and a stable high confinement state is entered. As the input power 


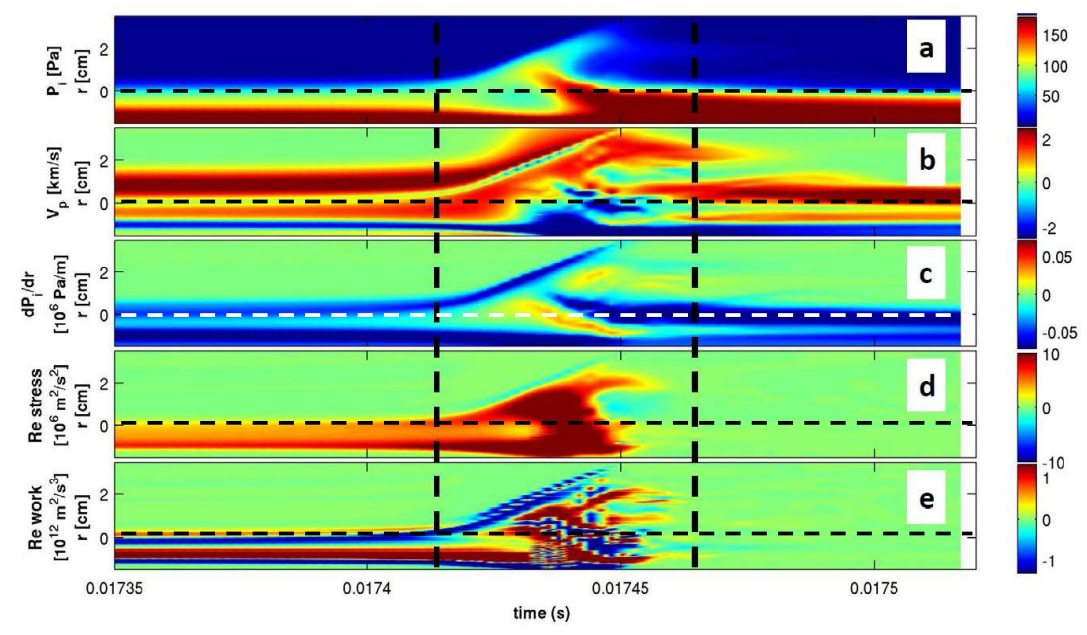

Figure 6. Last cycle of the I-phase from HESEL Fig 5. a) ion pressure, b) poloidal velocity, c) ion pressure gradient, d) Reynolds stress, e) Reynolds work: $<\tilde{v}_{r} \tilde{v}_{p}>\partial V_{p} / \partial r$. The horizontal dashed lines mark the LCFS $(r=0)$. The frames do not contain the inner region, where profiles are forced.

increases the length of the dithering cycles increases in line with building up the ion pressure gradient and mean flow, until the system is able to enter the H-mode. The quiet phases during the I-phase thus resemble the H-mode, with the MF not yet strong enough to fully sustain it. The evolution after the last cycle starts up with a quiet phase, which is now sufficiently supported to stay in a quiet phase and do not run away by being disrupted by the turbulence burst. We observe in Fig. 6e that the Re-work has a very complex radial structure with different signs at different radial positions. Considering the integral flow energy $V_{0}=\int \frac{1}{2} V_{p}^{2} d r$ we can show

$$
\frac{d V_{0}}{d t}=\int W_{R e} d r-\text { dissipation terms }+O T
$$

Thus, the Re work will act as decreasing the flow in regions where it is negative and increase the flow in regions where it is positive. We should note, however, that for the HESEL model with full ion dynamics, there may be other terms $(O T)$ arising from the correlation of the ion and electron pressure fluctuations that add directly to the flow production. This will be investigated in detail elsewhere [50].

The details of the LCO is furthermore described in Fig. 7, which displays the phase space diagram of the pressure vs. the poloidal velocity at different radial positions Lissajous diagrams. The three phases described above are marked by different colors red, green, and blue. We clearly see the LCO nature in the phase diagrams, but we also note that the direction of the "flow" in the phase diagram is different for the different radial positions. This is indicative for a radial propagation of the turbulence in the burst phases, which has been observed also in experiments, see, e.g., [16] and [17]. 

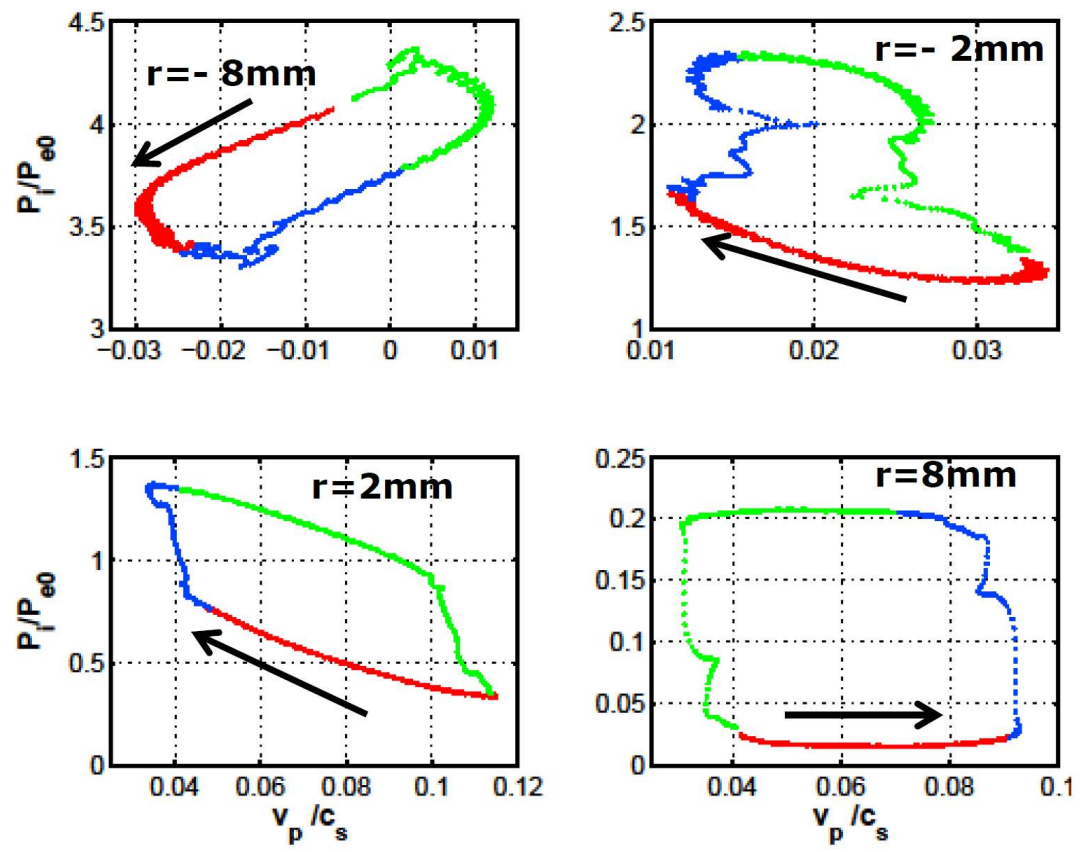

Figure 7. Lissajous diagrams of the pressure evolution versus the poloidal velocity variation at different radial positions.

\section{Discussion}

We have presented results from a numerical modeling of the $\mathrm{L}-\mathrm{H}$ transition in magnetically confined plasma. The investigations are based on the HESEL fluid model, which uniquely includes consistent evolution of profiles, edge-SOL coupling, ion energy dynamics, and collision and neo-classical transport parameters determined consistently from experimentally realistic plasma parameters. By considering a 2D model, contrary to the 3D models applied in other simulations, it was possible to run the code with realistic parameters over realistic long time spans of $10^{7} \omega_{c i}^{-1}$ and covering $10^{4}$ ion collision times. The model reproduces essential features of $\mathrm{L}-\mathrm{H}$ transitions of various types ranging from one-step fast transitions to slow L-I-H transitions with an intermediate phase characterized by limit cycle oscillations (LCO). We have obtained scalings of the power threshold that agrees with the high density branch of the commonly observed density scaling. Additionally, it was observed that the SOL loss dynamics plays an important role for the power threshold. A short parallel connection length leading to a short SOL depletion time leads to a lower power threshold indicating that double null divertor configurations have lower threshold than single null configurations.

The model appears to include the necessary and essential ingredients for the transition behavior, but in order to be fully predictive the model should naturally be improved in several respects: inclusion of toroidal geometry effects, electromagnetic effects, neutral particle interactions and plasma sources, general and realistic power input schemes ect. However, the results presented here should be seen as an essential step connecting the zero- and one-dimensional heuristic transition models with a predictive 
model and thus the full set of toroidal plasma dynamics. The ITER experiment, which relies on controlled H-mode access, will strongly need this gap in understanding to be bridged.

\section{Acknowledgments}

This work was supported by the National Magnetic Confinement Fusion Science Program of China under Contracts No. 2011GB107001, by National Natural Science Foundation of China under Contracts No. 11422546 and the Sino-Danish Center for Education and Research (SDC).

\section{References}

[1] Wagner F et al 1982 Phys. Rev. Lett., 491408.

[2] Garcia O E et al. 2006 Plasma Phys. Controlled Fusion, 48, L1.

[3] Naulin V 2007 J. Nucl. Mater., 363-365, 24.

[4] Wagner F 2007 Plasma Phys. Controlled Fusion, 49, B1.

[5] Moyer R A et al. 1995 Phys. Plasmas, 2, 2397.

[6] Cziegler I et al. 2014 Plasma Phys. Controlled Fusion, 56, 075013.

[7] Tynan G et al. 2015 Contribution in this issue.

[8] Zohm H et al. 1994 Phys. Rev. Lett., 72, 222.

[9] Xu G S et al. 2011 Phys. Rev. Lett., 107, 125001.

[10] Xu G S et al. 2014 Nucl. Fusion, 54, 013007.

[11] Xu G S et al. 2014 Nucl. Fusion, 54, 103002.

[12] Schmitz, L et al. 2012 Phys. Rev. Lett., 108, 155002.

[13] Schmitz, L et al. 2014 Nucl. Fusion, 54, 073012.

[14] Cheng J et al. 2013 Phys. Rev. Lett., 110, 265002.

[15] Kobayashi T et al. 2013 Phys. Rev. Lett., 111, 035002.

[16] Kobayashi T et al. 2014 Nucl. Fusion, 54, 073017.

[17] Estrada T eta al. 2015 Nucl. Fusion, 55063005.

[18] Kim E J and Diamond P H. 2003 Phys. Rev. Lett., 90, 185006.

[19] Dam M et al. 2013 Phys. Plasmas, 20, 102302.

[20] Miki K et al. 2012 Phys. Plasmas, 19, 092306.

[21] Wu X et al. 2015 Nucl. Fusion, 55, 053029.

[22] Malkov M A et al. 2015 Phys. Plasmas, 22, 032506.

[23] Ryter F et al. 2014 Nucl. Fusion, 54, 083003. See also contribution in this issue.

[24] Rogers B N, Drake J F, and Zeiler A 1998 Phys. Rev. Lett., 81 , 4396.

[25] Xu X Q et al. 2000 Phys. Plasmas, 7, 1951.

[26] Thyagaraja A, Valovic M, and Knight P J 2010 Phys. Plasmas, 17, 042507.

[27] Chone L et al. 2014 Phys. Plasmas, 21, 070702.

[28] Sarazin Y et al. 2000 Physica Scripta T, 84, 14.

[29] Garcia O E et al. 2007 J. Nucl. Mater., 363-365 575.

[30] Nielsen A H eta al. 2013 in Conference Paper 40th European Physical Society Conference on Plasma Physics, Espoo, Finland, webpage http://ocs.ciemat.es/EPS2013PAP/pdf/P5.173.pdf (2013).

[31] Madsen J et al. 2015 In preparation.

[32] Braginskii S I 1965 In Review of Plasma Physics, (ed. Leontovich, M. A.). New York, NY: Consultants Bureau, Vol. 1, p. 205.

[33] Garcia O E et al. 2005 Phys. Plasmas, 12, 062309. 
[34] Fundamenski W et al. 2007 Nucl. Fusion, 47, 417.

[35] Militello F et al. 2013 Plasma Phys. Controlled Fusion, 55, 025005.

[36] Yan N et al. 2013 Plasma Phys. Controlled Fusion, 55, 115007.

[37] Hinton F L and Horton C W 1971 Phys. Fluids, 14, 116.

[38] Naulin V and Nielsen A H 2003 SIAM J. Sci. Comput. 25, 104.

[39] Guzdar P N et al. 1993 Phys. Fluids B, 5, 3712.

[40] Scott B D 2005 Phys. Plasmas, 12, 062314.

[41] Meyer H et al. 2005 Plasma Phys. Controlled Fusion, 47, 843.

[42] Meyer H et al. 2006 Nucl. Fusion, 46, 64.

[43] Maingi R et al. 2005 Nucl. Fusion, 45, 1066.

[44] Liu Z X et al. 2013 Nucl. Fusion, 54, 073041.

[45] Martin Y et al. 2014 Nucl. Fusion, 54, 114006.

[46] Meyer H et al. 2011 Nucl. Fusion, 51, 113011.

[47] Ma Y et al. 2012 Plasma Phys. Controlled Fusion, 54, 082002.

[48] Nielsen A H et al. 2015 Phys. Lett. A, in press.

[49] Liu S C et al. Rev. Sci. Instrum., 83, 123506.

[50] Madsen J et al. (2015) In preparation. 